ELECTRONIC RESEARCH ANNOUNCEMENTS OF THE AMERICAN MATHEMATICAL SOCIETY

Volume 12, Pages 56-62 (May 16, 2006)

S $1079-6762(06) 00160-0$

\title{
A NEW TOWER OF RANKIN-SELBERG INTEGRALS
}

\author{
DAVID GINZBURG AND JOSEPH HUNDLEY
}

(Communicated by Barry Mazur)

\begin{abstract}
We recall the notion of a tower of Rankin-Selberg integrals, and two known towers, making observations of how the integrals within a tower may be related to one another via formal manipulations, and offering a heuristic for how the $L$-functions should be related to one another when the integrals are related in this way. We then describe three new integrals in a tower on the group $E_{6}$, and find out which $L$-functions they represent. The heuristics also predict the existence of a fourth integral.
\end{abstract}

\section{INTRODUCTION}

The notion of a tower of Rankin-Selberg integrals was introduced in G-R. To recall this notion, let $G$ be a reductive group defined over a global field $F$. Let ${ }^{L} G$ denote the $L$-group of $G$. Let $\rho$ denote a finite-dimensional irreducible representation of ${ }^{L} G$. Given an irreducible generic cuspidal representation of $G(\mathbf{A})$, we let $L^{S}(\pi, \rho, s)$ denote the partial $L$-function associated with $\pi$ and $\rho$. Here $s$ is a complex variable, and $\mathbf{A}$ denotes the adèle ring associated with $F$. If $\rho$ acts on the vector space $V$, we denote by $\mathbf{C}[V]$ the symmetric algebra attached to the vector space $V$. Let $\mathbf{C}[V]^{L} G$ denote the ${ }^{L} G$-invariant polynomials inside the symmetric algebra. As far as we know, all the examples of $L$-functions represented by a Rankin-Selberg integral are associated with representations $\rho$ such that $\mathbf{C}[V]^{L} G$ is a free algebra. A list of all such groups, representations, and the degrees of the generators of the invariant polynomials are given in $[\mathrm{K}$.

The basic observation in $[\mathrm{G}-\mathrm{R}]$ is that there is some relation between the Eisenstein series one uses to construct the Rankin-Selberg integral and the number of generators of the invariant polynomials and their degrees. This relation is far from being clear, and it is mainly based on observation of all known constructions of such integrals. To summarize in an unprecise manner, the relations are:

1) If $\rho_{1}$ and $\rho_{2}$ have the same number of generators with the same degrees, then in some cases the Rankin-Selberg integrals that represent the corresponding two $L$-functions use the same Eisenstein series.

2) Suppose that the Eisenstein series one uses for a certain construction is defined on $H(\mathbf{A})$, where $H$ is a reductive group. Suppose that this Eisenstein series corresponds to an induced representation induced from a parabolic subgroup $P=M U$ of $H$. Here $M$ is the Levi part of $P$, and $U$ its unipotent radical. As in the work of Shahidi, the group ${ }^{L} M$ acts on ${ }^{L} U$ by conjugation, and this way one obtains $r$

Received by the editors October 12, 2005.

2000 Mathematics Subject Classification. Primary 32N10.

(C)2006 American Mathematical Society Reverts to public domain 28 years from publication 
irreducible finite-dimensional representations of ${ }^{L} M$. Suppose that the corresponding Rankin-Selberg integral represents the $L$-function $L^{S}(\pi, \rho, s)$. Let $k$ denote the number of generators of $\mathbf{C}[V]^{L} G$, where we recall that that $\mathbf{C}[V]^{L} G$ is assumed to be free. Then the second observation (cf. [G-R, p. 202]) is that $r \geq k$.

It should be stressed that these two observations are based mainly on experience and we are not aware of precise theoretical reasons. We also want to mention that information on $L$-functions $L^{S}(\pi, \rho, s)$ where $\rho$ does not satisfy the above properties can be obtained using other methods, such as lifting theory.

In this paper we wish to point out two more observations that may shed some more light on the above relations. It will be convenient to first illustrate these observations by two examples.

Consider the following example of a tower given in $\mathrm{G}-\mathrm{R}$ :

\begin{tabular}{|l|l|l|l|}
\hline & $G$ & ${ }^{L} G$ & $\rho$ \\
\hline$(\mathbf{a 1})$ & $G L_{n}$ & $G L_{n}(\mathbf{C})$ & $2 \varpi_{1}$ \\
$(\mathbf{a 2})$ & $G L_{n} \times G L_{n}$ & $G L_{n}(\mathbf{C}) \times G L_{n}(\mathbf{C})$ & $\varpi_{1} \times \varpi_{1}$ \\
$(\mathbf{a 3})$ & $G L_{2 n}$ & $G L_{2 n}(\mathbf{C})$ & $\varpi_{2}$ \\
\hline
\end{tabular}

We recall the construction of the Rankin-Selberg integral which represents the $L$-function in case (a3). This integral was introduced in [J-S] and is given by

$$
\int_{S_{1}} \int_{S_{2}} \varphi_{\pi}\left(\left(\begin{array}{cc}
I & X \\
& I
\end{array}\right)\left(\begin{array}{ll}
g & \\
& g
\end{array}\right)\right) E(g, s) \psi(\operatorname{tr} X) d X d g .
$$

Here $\varphi_{\pi}$ is a vector in the space of $\pi$ which is an irreducible cuspidal representation defined on $G L_{2 n}(\mathbf{A}), E(g, s)$ is an Eisenstein series defined on the group $G L_{n}(\mathbf{A})$, and $S_{1}=Z(\mathbf{A}) G L_{n}(F) \backslash G L_{n}(\mathbf{A}), S_{2}=M a t_{n \times n}(F) \backslash M a t_{n \times n}(\mathbf{A})$. For more details see [J-S]. Let us show how the integral that represents the $L$-function given in (a2) can be derived from integral (11). First notice that $G L_{n} \times G L_{n}$ is a Levi part of a maximal parabolic subgroup $P$ of $G L_{2 n}$. Now suppose we formally replace in (11) the cuspidal representation $\pi$ by the Eisenstein series $E_{\tau, \sigma}(g, \nu)$ associated with the induced representation $\operatorname{Ind}_{P(\mathbf{A})}^{G L_{2 n}(\mathbf{A})}(\tau \otimes \sigma) \delta_{P}^{\nu}$. Here $\tau$ and $\sigma$ are cuspidal representations defined on $G L_{n}(\mathbf{A})$, and $\nu$ is a complex variable. Of course the integral will not converge. However, if we ignore this issue, and formally unfold the Eisenstein series $E_{\tau, \sigma}(g, \nu)$, we are led to consider the space of double cosets

$$
P \backslash G L_{2 n} /\left\{\left(\begin{array}{cc}
g & X \\
g
\end{array}\right) \mid G \in G L_{n}, X \in M a t_{n \times n}\right\} .
$$

If we consider the open orbit contribution to the integral, it is not hard to check that we obtain the integral

$$
\int_{Z(\mathbf{A}) G L_{n}(F) \backslash G L_{n}(\mathbf{A})} \varphi_{\tau}(g) \varphi_{\sigma}(g) E(g, s) d g
$$

as inner integration. As is well known, integral (2) represents the tensor-product $L$-function of $\tau \times \sigma$. In other words, this integral is the one which represents the $L$-function described in case $(\mathbf{a 2})$. Furthermore, if one restricts the exterior square representation $\varpi_{2}^{\prime}$ of $G L_{2 n}(\mathbf{C})$ to $G L_{n}(\mathbf{C}) \times G L_{n}(\mathbf{C})$, then one obtains $\left.\varpi_{2}^{\prime}\right|_{G L_{n} \times G L_{n}}=\left(\varpi_{1} \times \varpi_{1}\right) \oplus\left(\varpi_{2} \times 1\right) \oplus\left(1 \times \varpi_{2}\right)$. From this we deduce the following. If we start with the representation $\rho$ as defined in case (a3) and restrict it to the $L$ group of the Levi part, then the representation $\rho$ corresponding to case (a2) occurs in the restriction. Moreover, it is the representation with the largest dimension which occurs in the restriction. 
The formal replacement of a cuspidal representation by an Eisenstein series and then an analysis of the contribution from the open orbit is one of the observations we wish to make. It should be mentioned that this observation does not explain how to derive a global construction that will represent the $L$-function described in case (a1).

We now consider the second example of a tower as described in $\mathrm{G}-\mathrm{R}$. This tower consists of four members as follows:

\begin{tabular}{|l|l|l|l|}
\hline & $G$ & ${ }^{L} G$ & $\rho$ \\
\hline (b1) & $G L_{2}$ & $G L_{2}(\mathbf{C})$ & $4 \varpi_{1}$ \\
(b2) & $G L_{3}$ & $G L_{3}(\mathbf{C})$ & $\varpi_{1}+\varpi_{2}$ \\
$(\mathbf{b 3})$ & $G S p i n_{7}$ & $G S p_{6}(\mathbf{C})$ & $\varpi_{2}$ \\
$(\mathbf{b 4})$ & $F_{4}$ & $F_{4}(\mathbf{C})$ & $\varpi_{4}$ \\
\hline
\end{tabular}

The construction of Rankin-Selberg integrals for cases (b1), (b3), and (b4) was given in G-R. Case (b2) was studied in G1. The integral which represents the $L$ function given in (b4) can be described as follows. Let $\pi$ denote a generic cuspidal representation defined on the group $F_{4}(\mathbf{A})$. Let $E(g, s)$ denote the degenerate Eisenstein series defined on the exceptional group $G_{2}(\mathbf{A})$ as described in G-R, Section 1]. The global integral is

$$
\int_{G_{2}(F) \backslash G_{2}(\mathbf{A})} \int_{U(F) \backslash U(\mathbf{A})} \varphi_{\pi}(u g) E(g, s) \psi_{U}(u) d u d g .
$$

Here $U$ is a certain unipotent subgroup of $F_{4}$, and $\psi_{U}$ is an additive character defined on the group $U$. Observe that $\operatorname{SSpin}_{7}$ is a Levi part of a maximal parabolic subgroup $P$ of $F_{4}$. Let $\tau$ denote a generic cuspidal representation defined on the group $\operatorname{GSpin}_{7}$. Let $E_{\tau}(g, \nu)$ denote the Eisenstein series defined on the group $F_{4}(\mathbf{A})$ and associated to the induced representation $\operatorname{Ind}_{P(\mathbf{A})}^{F_{4}(\mathbf{A})} \tau \delta_{P}^{\nu}$. If we formally replace in (3) the cuspidal representation $\pi$ by $E_{\tau}(g, \nu)$, and then unfold this Eisenstein series, then we obtain from the open orbit

$$
\int_{G_{2}(F) \backslash G_{2}(\mathbf{A})} \varphi_{\tau}(g) E(g, s) d g
$$

as inner integration. As described in [G-R, Section 4], this is precisely the global integral which represents the $L$-function described in (b3). Furthermore, let $Q$ denote the maximal parabolic subgroup of $\operatorname{Spin}_{7}$ whose Levi part is $G L_{3}$. Let $\sigma$ denote a cuspidal representation defined on the group $G L_{3}(\mathbf{A})$. Replace in (4) the cuspidal representation $\tau$ by the Eisenstein series $E_{\sigma}(g, \nu)$ associated with the induced representation $\operatorname{Ind}_{Q(\mathbf{A})}^{\operatorname{Spin}_{7}(\mathbf{A})} \sigma \delta_{Q}^{\nu}$. Unfolding the integral, we obtain from the open orbit

$$
\int_{S L_{3}(F) \backslash S L_{3}(\mathbf{A})} \varphi_{\sigma}(g) E(g, s) d g
$$

as inner integration. As described in [G1, this is precisely the global integral that represents the $L$-function described in (b2).

As in the previous case we can restrict in each case the representations $\rho$ to the $L$-group of the Levi part. All representations are labeled by $n$-tuples of integers corresponding to the coefficients of the fundamental weights, in the highest weight of that representation. This does not specify the action of the central torus, but is sufficient for our present purposes. The fundamental weights are numbered 
in the manner that is customary in the literature. Thus $(0,0,0,1)$ is the representation of $F_{4}(\mathbf{C})$ of dimension 26. This is the representation $\rho$ obtained in case (b4). Restrict it to $G S p_{6}(\mathbf{C})$, which is the $L$-group of $G S_{p i n}$. We obtain $\left.(0,0,0,1)\right|_{G S p_{6}}=(0,1,0)+2(1,0,0)$. Here $(0,1,0)$ is the second fundamental representation of $G S p_{6}(\mathbf{C})$, which has degree 14 , and $(1,0,0)$ is the six-dimensional standard representation. If we further restrict $G S p_{6}(\mathbf{C})$ to $G L_{3}(\mathbf{C})$, we obtain $\left.(0,1,0)\right|_{G L_{3}}=(1,1)+(1,0)+(0,1)$. Again, as in the first tower, we can see that if we restrict $\rho$ as defined in case (b4), we obtain the representation $\rho$ as defined in case (b3) as the largest piece in the restriction. A similar relationship exists between cases (b3) and (b2).

We mention again that this observation does not allow one to obtain the integrals for cases (a1) and (b1). The construction in these cases is more complicated and involves covering groups.

To summarize, the above examples suggest the following two points.

3) Suppose that we are given a Rankin-Selberg integral which we know how to unfold to an Eulerian integral with the Whittaker function defined on the cuspidal representations. Then replacing a cuspidal representation by an Eisenstein series and considering the contribution from the open orbit sometimes yield a new Eulerian Rankin-Selberg integral. In fact, one can replace the cuspidal representation by various Eisenstein series. Experience indicates that most of the time one gets either zero, or an integral which does not unfold to a Whittaker integral.

4) Suppose that the Eulerian integral with which we start represents an $L$ function associated to the finite-dimensional irreducible representation $\rho$ of the complex group ${ }^{L} G$. Suppose that we replace a cuspidal representation, defined over the group $G(\mathbf{A})$, by an Eisenstein series induced from a cuspidal representation defined on the Levi part $M(\mathbf{A})$. Suppose that when we formally unfold the new integral, the contribution from the open orbit produces a new integral which is Eulerian with Whittaker functions. Then the new integral will represent the $L$ function associated with the largest irreducible representation that occurs in the restriction $\left.\rho\right|_{L_{M}}$.

In these notes we announce a construction of a new tower of Rankin-Selberg integrals. The tower we consider is the following:

\begin{tabular}{|l|l|l|l|}
\hline & $G$ & ${ }^{L} G$ & $\rho$ \\
\hline$(\mathbf{c 1})$ & $G L_{3} \times G L_{2}$ & $G L_{3}(\mathbf{C}) \times G L_{2}(\mathbf{C})$ & $2 \varpi_{1} \times \varpi_{1}$ \\
$(\mathbf{c 2})$ & $G L_{3} \times G L_{3} \times G L_{2}$ & $G L_{3}(\mathbf{C}) \times G L_{3}(\mathbf{C}) \times G L_{2}(\mathbf{C})$ & $\varpi_{1} \times \varpi_{1} \times \varpi_{1}$ \\
$(\mathbf{c 3})$ & $G L_{6} \times G L_{2}$ & $G L_{6}(\mathbf{C}) \times G L_{2}(\mathbf{C})$ & $\varpi_{2} \times \varpi_{1}$ \\
$(\mathbf{c 4})$ & $E_{6} \times G L_{2}$ & $E_{6}(\mathbf{C}) \times G L_{2}(\mathbf{C})$ & $\varpi_{1} \times \varpi_{1}$ \\
\hline
\end{tabular}

It follows from $[\mathrm{K}]$ that in all these representations the ${ }^{L} G$-invariant algebra has one generator of degree 12. At this point we know a Rankin-Selberg construction for all three cases $(\mathbf{c 2})-(\mathbf{c 4})$. In the next section we shall explain these constructions and show on an example how to derive one integral from the other. One can also check that restricting from one case to the other does indeed produce the right representation $\rho$ in each case.

It should be mentioned that all of these $L$-functions can be studied using the Langlands-Shahidi method as explained in $[\mathrm{S}]$. 


\section{The GLOBAL INTEGRALS}

We start with the global construction which will correspond to case (c4), as explained in the Introduction. Let $G$ denote the exceptional similitude group of type $E_{6}$, constructed exactly as in G2]. To introduce the global integral we need to consider two small representations which we now define. First, let $\theta$ denote the minimal representation defined on $G(\mathbf{A})$. This representation was constructed and studied in G-R-S. The construction there is defined on the group $E_{6}$; however, this definition can be extended to similitude groups without any problem. See G-J] for a similar definition of the exceptional similitude group $G E_{7}$. In this paper we denote a function in the space of this representation by $\theta(g)$. Another representation we need for our construction was defined and studied in $\mathrm{G}-\mathrm{H}$, Section 3]. The representation constructed there was defined on the group $G S O_{10}(\mathbf{A})$. A similar definition holds for the group $\operatorname{GSpin}_{10}(\mathbf{A})$. This representation depends on a cuspidal representation $\tau$ defined on $G L_{2}(\mathbf{A})$, with trivial central character. We shall denote a vector in this space by $\theta_{\tau}(h)$, where $h \in G \operatorname{Spin}_{10}(\mathbf{A})$. We briefly recall the definition. Let $R$ denote the parabolic subgroup of $G$ Spin $_{10}$ whose Levi part is $G L_{3} \times G \operatorname{Spin}_{4}$. Let $\mu(\tau)$ denote the symmetric square lift of $\tau$ to $G L_{3}$ as constructed in Ge-J, and let $\epsilon(\tau)$ denote the outer tensor product $\tau \otimes \tau$. A priori, this is a representation of $P G L_{2} \times P G L_{2}$, but $G S p i n_{4}$ covers this group. Let $E(\tau, h, s)$ denote the Eisenstein series defined on $\operatorname{GSpin}_{10}(\mathbf{A})$ and associated with the induced representation $\operatorname{Ind}_{R(\mathbf{A})}^{G \operatorname{Spin}_{10}(\mathbf{A})}(\mu(\tau) \otimes \epsilon(\tau)) \delta_{R}^{s}$. It is not hard to check that this Eisenstein series has a unique pole in the domain $\operatorname{Re}(s)>\frac{1}{2}$, which is simple and located at $s=2 / 3$. We denote the residue representation by $\theta_{\tau}$.

Using this last representation, we now construct the Eisenstein series we use in our global construction. Let $P$ denote the maximal standard parabolic subgroup of $G$ whose Levi part contains all the simple roots except $\alpha_{1}$. This Levi part is essentially $G \operatorname{Spin}_{10}$. Let $E_{\tau}(g, s)$ denote the Eisenstein series defined on $G(\mathbf{A})$ and associated to the induced representation $\operatorname{Ind}_{P(\mathbf{A})}^{G(\mathbf{A})} \theta_{\tau} \delta_{P}^{s}$.

Let $\pi$ denote a generic cuspidal representation defined on $G(\mathbf{A})$. We assume that $\pi$ has a trivial central character. Consider the global integral

$$
\int_{Z(\mathbf{A}) G(F) \backslash G(\mathbf{A})} \varphi_{\pi}(g) \theta(g) E_{\tau}(g, s) d g .
$$

Here $Z$ denotes the center of $G$, and $\varphi_{\pi}$ is a cusp form in the space of $\pi$. This integral represents the $L$-function corresponding to case (c4).

Let us show how to obtain the Rankin-Selberg integral which will represent case (c3) as denoted in the Introduction. Let $Q$ denote the maximal parabolic subgroup of $G$ whose Levi part is $M=G L_{1} \times G L_{6}$. Let $\sigma$ denote a cuspidal representation of $G L_{6}(\mathbf{A})$ with trivial central character. Let $E_{\sigma}(g, \nu)$ denote the Eisenstein series defined on $G(\mathbf{A})$ and associated with the induced representation $\operatorname{Ind}_{Q(\mathbf{A})}^{G(\mathbf{A})} \sigma \delta_{Q}^{\nu}$. In (6) we replace the function $\varphi_{\pi}(g)$ by $E_{\sigma}(g, \nu)$. Even though the integral does not converge, we formally unfold the Eisenstein series $E_{\sigma}(g, \nu)$ to obtain

$$
\int_{Z(\mathbf{A}) M(F) U(F) \backslash G(\mathbf{A})} f_{\sigma}(g, \nu) \theta(g) E_{\tau}(g, s) d g .
$$

Here $U$ is the unipotent radical of $Q$, and $f_{\sigma}(g, \nu)$ defines a section in the corresponding induced representation. Recall that $U$ has a structure of a Heisenberg 
group with 21 variables. Let $x_{122321}(r)$ denote the one-dimensional unipotent subgroup that is the center of $U$. Here, and henceforth we shall use the notation for various roots of the group $G$ as defined in G2]. We expand $\theta(g)$ along the center of $U$. That is, we expand it along the unipotent group generated by $x_{122321}(r)$ with points in $F \backslash \mathbf{A}$. The group $M(F)$ acts on this expansion with two orbits. Ignoring the trivial orbit, we obtain the contribution

$$
\int_{Z(\mathbf{A}) H(F) U(F) \backslash G(\mathbf{A})} \int_{F \backslash \mathbf{A}} \theta\left(x_{122321}\left(r_{1}\right) g\right) \psi\left(r_{1}\right) d r_{1} f_{\sigma}(g, \nu) E_{\tau}(g, s) d g .
$$

Here $H$ is the stabilizer inside $M$ of the character $\psi$. One can check that $H=\{g \in$ $G L_{6}: \operatorname{det} g$ is a square\}. Factoring the integration over $H$ and over the center of $U$, we obtain, after a change of variables, the integral

$$
\int_{S_{1}} \int_{S_{2}} \int_{S_{3}} \varphi_{\sigma}(h) \theta\left(u x_{122321}\left(r_{1}\right) h\right) E_{\tau}\left(u x_{122321}\left(r_{2}\right) h, s\right) \psi\left(r_{1}-r_{2}\right) d r_{1} d r_{2} d h
$$

as inner integration. Here $\varphi_{\sigma}$ is a cusp form in the space of the cuspidal representation $\sigma, S_{1}=Z(\mathbf{A}) H(F) \backslash H(\mathbf{A}), S_{2}=U(F) \backslash U(\mathbf{A}), S_{3}=(F \backslash \mathbf{A})^{2}$. Notice that this integral converges absolutely. This is the Rankin-Selberg integral that represents case (c3).

We can continue further and replace $\sigma$ by an Eisenstein series. Indeed, let $\pi_{1}$ and $\pi_{2}$ denote two cuspidal representations of $G L_{3}(\mathbf{A})$. Let $L$ denote the parabolic subgroup of $G L_{6}$ whose Levi part is $G L_{3} \times G L_{3}$. Let $E_{\pi_{1}, \pi_{2}}(x, \nu)$ denote the Eisenstein series associated with the induced representation $\operatorname{Ind}_{L(\mathbf{A})}^{G L_{6}(\mathbf{A})}\left(\pi_{1} \otimes \pi_{2}\right) \delta_{L}^{\nu}$. Replacing in (91) the cuspidal representation $\sigma$ by this Eisenstein series (again, this is a formal process, since the integral does not converge) and performing certain Fourier expansions, one obtains the integral

$$
\begin{array}{r}
\int_{Z(\mathbf{A}) H(F) \backslash H(\mathbf{A})} \int_{V(F) \backslash V(\mathbf{A})} \int_{(F \backslash \mathbf{A})^{3}} \varphi_{\pi_{1}, \pi_{2}}(h) \theta\left(x_{010000}\left(r_{1}\right) v x_{112321}\left(r_{2}\right) x_{122321}\left(r_{3}\right) h\right) \\
\times E_{\tau}\left(x_{010000}\left(r_{1}\right) v h, s\right) \psi\left(r_{1}+r_{2}\right) d r_{i} d v d h
\end{array}
$$

as inner integration. Here $\varphi_{\pi_{1}, \pi_{2}}$ is a vector in the space of $\pi_{1} \otimes \pi_{2}$. We also have $H=\left\{\left(g_{1}, g_{2}\right) \in G L_{3} \times G L_{3}: \operatorname{det} g_{1}=\operatorname{det} g_{2}\right\}$, and the group $V$ is the standard unipotent radical of the maximal parabolic subgroup of $G$ whose Levi part is $G L_{3} \times G L_{3} \times G L_{2}$. This is the integral that represents case (c2).

At this point we unfolded all these three integrals and established that they are indeed Eulerian. This we achieved by showing that once the integrals are unfolded, the resulting integrals involve functions in the Whittaker models of the cuspidal representations, and hence are Eulerian by the usual uniqueness of the model. As always, with these type of integrals, the unfolding process is long and tedious but quite straightforward. The next step is to compute the unramified local integrals. We describe this process briefly. First, the unramified local integral is expressed as a power series in $q^{-s}$, with coefficients being the characters of finite-dimensional representations of the $L$-group, evaluated at the Satake parameter of the cuspidal representation that appeared in the original integral. A local $L$-function is a similar object: if $t_{\pi}$ is our Satake parameter and $\rho$ is our representation of the $L$-group, then the coefficient of $q^{-n s}$ is $\operatorname{Tr}\left(\operatorname{Sym}^{n} \rho\left(t_{\pi}\right)\right)$. We are thus reduced to proving an identity in the ring $R\left[{ }^{L} G\right][[X]]$ of formal power series over the representation 
ring of the $L$-group, which amounts to a description of the decomposition of the symmetric algebra of the original representation. It is not yet clear to us how complicated the decomposition of the symmetric algebras will be in various cases. It will also be interesting to study the possible poles of these $L$-functions. This will be accomplished by understanding the poles of the Eisenstein series we use in all these cases.

We are also interested in finding the Rankin-Selberg integral which represents case $(\mathbf{c 1})$. Past experience indicates that some of the representations involved should be defined on a covering group. So far we do not know how to do it.

We summarize.

Theorem. Integrals (6), (9), and (10) are Eulerian. Each of these integrals unfolds to an Eulerian integral involving the Whittaker model of each cuspidal representation appearing in the original integral. Integral (6) represents the partial $L$-function $L^{S}(\pi \times \tau, S t \times S t, s)$, where $S t \times S t$ corresponds to the standard representation of $E_{6}(\mathbf{C}) \times G L_{2}(\mathbf{C})$. Integral (9) represents $L^{S}\left(\sigma \times \tau, \wedge^{2} \times S t, s\right)$, where $\wedge^{2}$ is the exterior square representation of $G L_{6}(\mathbf{C})$, and integral (10) represents $L^{S}\left(\pi_{1} \times \pi_{2} \times \tau, S t \times S t \times S t, s\right)$.

\section{REFERENCES}

[Ge-J] S. Gelbart, H. Jacquet, A relation between automorphic representations of GL(2) and GL(3). Ann. Sci. Ecole Norm. Sup. (4) 11 (1978), no. 4, 471-542. MR0533066|(81e:10025)

[G1] D. Ginzburg, A Rankin-Selberg integral for the adjoint representation of $G L_{3}$, Invent. Math. 105 (1991), 571-588. MR.1117151 (92f:11070)

[G2] D. Ginzburg, On standard $L$-functions for $E_{6}$ and $E_{7}$. J. Reine Angew. Math. 465 (1995), 101-131. MR1344132 (96m:11040)

[G-H] D. Ginzburg, J. Hundley, On spin $L$-functions for $G S O_{10}$. To appear in J. Reine Angew. Math. ArXiv:math.NT/0512113.

[G-J] D. Ginzburg, D. Jiang, Periods and liftings: From $G_{2}$ to $C_{3}$. Israel J. of Math. 123 (2001), 29-59. MR:1835288 (2002f:11054)

[G-R] D. Ginzburg, S. Rallis, A tower of Rankin-Selberg integrals. IMRN 5 (1994), 201-208. MR1270133 (96a:11047)

[G-R-S] D. Ginzburg, S. Rallis, D. Soudry, On the automorphic theta representation for simply laced groups. Israel J. of Math. 100 (1997), 61-116. MR.1469105 (99c:11058)

[J-S] H. Jacquet, J. Shalika, Exterior square $L$-functions, automorphic forms, Shimura varieties, and $L$-functions, Vol. 2. Academic Press, 1990, pp. 143-226. MR.1044830 (91g:11050)

[K] V. Kac, Some remarks on nilpotent orbits. J. of Algebra 64 (1980), 190-213. MR 0575790 (81i:17005)

[S] F. Shahidi, On certain L-functions. Amer. J. Math. 103 (1981), no. 2, 297-355. MR0610479(82i:10030)

School of Mathematical Sciences, Sackler Faculty of Exact Sciences, Tel-Aviv UniVERSITY, TEL-AVIV 69978, IsRAEL

E-mail address: ginzburg@post.tau.ac.il

Mathematics Department, Penn State University, University Park, PA 16802

E-mail address: hundley@math.psu.edu 